\title{
(2) OPEN ACCESS \\ Comparison of macular structural and vascular changes in neuromyelitis optica spectrum disorder and primary open angle glaucoma: a cross- sectional study
}

\author{
Xiayin Zhang, ${ }^{1}$ Hui Xiao, ${ }^{1}$ Chunxin Liu, ${ }^{2}$ Lanqin Zhao, ${ }^{1}$ Jinghui Wang, ${ }^{1}$ Haiquan $\mathrm{Li}^{2}{ }^{2}$ \\ Ruixin Wang, ${ }^{1} \mathrm{Yi}_{\mathrm{Z}} \mathrm{Zhu},{ }^{3}$ Chuan Chen, ${ }^{4}$ Xiaohang Wu, ${ }^{1}$ Duoru Lin, ${ }^{1}$ Jingqi Wang, ${ }^{2}$ \\ Xing Liu, ${ }^{1}$ Wei Qiu, ${ }^{2}$ Patrick Yu-Wai-Man, ${ }^{5,6,7,8}$ Daniel S Ting (1) , ${ }^{1,9}$ Haotian Lin (1) 1,10
}

\begin{abstract}
- Additional material is published online only. To view please visit the journal online (http://dx.doi.org/10.1136/ bjophthalmol-2020-315842).

For numbered affiliations see end of article.
\end{abstract}

\section{Correspondence to}

Professor Haotian Lin, Zhongshan Ophthalmic Center, Sun Yat-sen University, Guangzhou, Guangdong, China; gddlht@aliyun.com

$\mathrm{XZ}, \mathrm{HX}, \mathrm{CL}$ and $\mathrm{LZ}$ are joint first authors.

$\mathrm{XL}, \mathrm{WQ}, \mathrm{PY}-\mathrm{W}-\mathrm{M}$ and DST are joint senior authors.

Received 8 January 2020 Revised 21 April 2020 Accepted 23 April 2020 Published Online First 19 May 2020

\begin{abstract}
Aims To compare macular structure and vasculature between neuromyelitis optica spectrum disorder (NMOSD) and primary open angle glaucoma (POAG) using optical coherence tomography angiography. Methods NMOSD patients $(n=124)$ with/without a history of optic neuritis (ON) (NMO+ON: 113 eyes; NMOON: 95 eyes), glaucomatous patients $(n=102)$ with early/ advanced glaucoma (G-E: 74 eyes; G-A: 50 eyes) and healthy controls ( $n=62 ; 90$ eyes) were imaged. The main outcome measures were macular ganglion cell-inner plexiform layer (GC-IPL) thickness, vessel density (VD) and perfusion density (PD) in the superficial capillary plexus, and diagnostic capabilities of the parameters as calculated by area under the curve (AUC).

Results Significant losses in GC-IPL, VD and PD were detected in both patients with NMOSD and POAG. With matched losses in the peripapillary retinal nerve fibre layer, NMOSD group showed significant thinning of GC-IPL in the nasal-superior quadrant, whereas in POAG group, significant thinning was observed in the inferior and temporal-inferior quadrants. GC-IPL thinning was more prominent in the superior, nasal-superior and nasal-inferior quadrants in $\mathrm{NMO}+\mathrm{ON}$ eyes. In G-A eyes, significant GC-IPL thinning was seen in the temporalinferior quadrant. The specific structural parameters combining VD and foveal avascular zone (FAZ) indices showed the best diagnostic accuracies. The FAZ area in eyes with NMOSD was significantly smaller than the eyes of healthy controls and POAG.
\end{abstract}

Conclusion NMOSD and POAG have specific patterns of macular structural and vascular changes associated with pathophysiology. Our results indicate that FAZ could be a sensitive biomarker of macular changes in NMOSD.

\section{INTRODUCTION}

Neuromyelitis optica spectrum disorder (NMOSD) is an autoimmune inflammatory disease of the central nervous system with a prevalence of 0.5-4.4 cases per 100000 people, which is characterised by severe attacks of recurrent optic neuritis (ON) and longitudinally extensive transverse myelitis. ${ }^{1}$ NMOSD carries a poor visual prognosis and patients often suffer from recurrent episodes of ON, the severity of which is strongly correlated with the amount of retinal atrophy caused by cumulative
ON episodes. ${ }^{3}$ The most common retinal abnormalities on optical coherence tomography (OCT) assessment of NMOSD are thinning of the peripapillary retinal nerve fibre layer ( $\mathrm{pRNFL}$ ) and the macular ganglion cell-inner plexiform layer (GCIPL).$^{5-7}$ Glaucoma affects more than 70 million people worldwide, with more than $80 \%$ of cases being primary open-angle glaucoma (POAG). This progressive optic neuropathy is also characterised by degeneration of the pRNFL and GC-IPL on OCT imaging. 89

Several studies have investigated the patterns of retinal ganglion cell loss in NMOSD or POAG compared with healthy controls. ${ }^{3}$ 10-12 The pRNFL in NMOSD was found to be particularly thin in the inferior and superior quadrants, as evaluated by four studies independently, ${ }^{10}$ and a similar pattern has been observed in POAG. ${ }^{8}$ In addition, an increased cup-disc ratio $(C / D)$, which is one of the distinguishing characteristics of POAG, has also been reported in patients with NMOSD. ${ }^{12} 13$ While the pRNFL comprises axons originating from ganglion cell neurons and reveals optic nerve status, macular GC-IPL is a direct reflection of the intrinsic ganglion cell bodies, which can provide information about primary retinal pathology. ${ }^{3}$ Macular GC-IPL measurements are more specific for detecting axonal loss than pRNFL measurements because macular parameters are not affected by optic nerve head oedema and are directly associated with visual outcomes. ${ }^{14} 15$ Currently, it is unclear whether the topographic changes in the macular GC-IPL and microvasculature are the same in NMOSD and POAG eyes.

Optical coherence tomography angiography (OCTA) techniques that non-invasively provide quantitative information on optic nerve structure and retinal vascular flow are likely to prove useful for specialists dealing with NMOSD and POAG, with the advantages of short acquisition times and sensitive measurements. ${ }^{16}{ }^{17}$ In this study, we used OCTA to define specific damage patterns in the macular GC-IPL and microvasculature, and to compare the patterns in NMOSD with those in POAG. The diagnostic accuracies for each group and subgroup (NMOSD with or without $\mathrm{ON}$ : $\mathrm{NMO}+\mathrm{ON}, \mathrm{NMO}-\mathrm{ON}$; glaucoma, early or advanced: G-E, G-A) were compared using different OCTA parameters. 


\section{MATERIALS AND METHODS}

\section{Subject recruitment}

Participants were volunteers recruited from Zhongshan Ophthalmic Center and Department of Neurology of Third Affiliated Hospital, Sun Yat-sen University, Guangzhou, China. Written informed consent was obtained from all participants. Exclusion criteria for all patients and the healthy controls included the following: (1) subjects diagnosed with other systemic diseases; (2) high myopia or hyperopia (spherical equivalent refractive error greater than -6 diopters or +3 diopters); (3) significant media opacity; (4) age $<18$ years old; and (5) no definite diagnosis of NMOSD or POAG.

Two neurologists confirmed the NMOSD diagnosis according to the 2015 revised diagnostic criteria. ${ }^{18}$ Patients with an episode of $\mathrm{ON}$ within the last 6 months were excluded to minimise the effect of optic disc swelling. Patients with previous ocular surgeries or POAG were also excluded.

Two glaucoma subspecialty trained ophthalmologists independently determined the presence of G-E and G-A. The POAG inclusion criteria were open angles on gonioscopy, with glaucomatous features of optic nerve neuropathy and/or reliable and repeatable visual field defects. G-E was defined as POAG eyes with reliable and repeatable glaucomatous visual field damage (mean deviation $\geq-6 \mathrm{~dB}$ ). G-A was defined as POAG eyes with reliable and repeatable glaucomatous visual field damage (mean deviation $<-6 \mathrm{~dB})$.

The criteria for inclusion in the healthy control group were no family history of NMOSD or POAG, intraocular pressure $<21 \mathrm{mmHg}$ with no history of elevated intraocular pressure, normal-appearing optic disc, intact neuroretinal rim and pRNFL and normal visual fields.

\section{Examinations}

All participants underwent an extensive ophthalmologic examination, including best-corrected visual acuity (BCVA), slit-lamp biomicroscopy, gonioscopy, intraocular pressure measurement with Goldmann applanation tonometry, dilated fundus examination, simultaneous stereo-photography of the optic disc, visual field testing by standard automated perimetry (SAP, Humphrey Field Analyser; 30-2 Swedish interactive threshold algorithm; Carl Zeiss Meditec, Jena, Germany) and OCTA scans (Cirrus 5000, V.10.0; Zeiss Meditec, California, USA). All patients with NMOSD were tested with MRI examinations and AQP4-IgG using commercially available cell-based assays (EUROIMMUN). ${ }^{19}$

\section{OCTA acquisition and processing}

OCTA imaging was performed using the high-definition OCT and AngioPlex device (Cirrus 5000, V.10.0; Zeiss Meditec, California, USA) with a wavelength of $840 \mathrm{~nm}$ and an A-scan rate of 68000 scans per second. To minimise patient-related motion, participants in this study were instructed to focus their gaze on the centre of the cross target and remain as still as possible during acquisition of the OCTA scans. Minimal blinking was permitted only after each scan to keep the cornea lubricated, and moderate amount of artificial tears were used in cases when the participants' eyes showed signs of drying out.

An optic disc cube $200 \times 200$ scan protocol was used for pRNFL measurements and the vertical C/D. pRNFL thickness values were measured for $3.46 \mathrm{~mm}$ diameter circles around the optic disc, pRNFL thickness parameters evaluated were average thickness and the thicknesses of four quadrant sectors (superior, temporal, inferior and nasal). A macular cube with
$512 \times 128$ scan mode was used for GC-IPL thickness measurements, within a $6 \mathrm{~mm}$ diameter centred at the fovea. The GC-IPL thickness parameters evaluated were average thickness of the $6 \mathrm{~mm}$ diameter region and the six quadrant sectors (superior, temporal-superior, temporal-inferior, inferior, nasal-inferior and nasal-superior).

Angiography imaging was conducted centred at the macula with the $6 \times 6 \mathrm{~mm}$ scan pattern. All scans were analysed using Cirrus OCTA software (AngioPlex, V.10.0; Carl Zeiss Meditec). $\mathrm{VD}$ is the total length of perfused vasculature per unit area in a region of measurement, while PD is the total area of perfused vasculature per unit area in a region of measurement. VD and PD were performed on the annular zone after excluding the foveal avascular zone (FAZ). The VD, PD and FAZ area of the superficial capillary plexus (from the internal limiting membrane to the inner plexiform layer) were automatically measured by software from Carl Zeiss Meditec using optical microangiography algorithms. The central foveal region was a region with a diameter of $1 \mathrm{~mm}$, and the inner and outer rings had outer diameters of 3 and $6 \mathrm{~mm}$ and were divided into four quadrants. The VD and PD values of nine quadrant sectors (central, superiorinner, temporal-inner, inferior-inner, nasal-inner, superior-outer, temporal-outer, inferior-outer and nasal-outer) and the whole area were analysed. Structural and microvasculature measurements in representative eyes are shown in figure 1.

Although eye movement is significantly reduced by asking the subjects to fixate on a target during OCTA imaging, microsaccades could not be avoided altogether. In this study, we assume that we could temporarily ignore the effect of motion artefact in the clinical comparison of multiple diseases by using the FastTrac retinal-tracking technology of high-definition OCT and the AngioPlex device (Cirrus 5000, V.10.0; Zeiss Meditec, California, USA) and strictly applying the established standards for OCTA image processing. An absence of motion artefacts was defined as no vessel doubling, vessel discontinuity/misalignment or lateral vessel displacement in the OCTA image. Images with a signal strength $<7$ and those with poor centration or segmentation errors were also excluded from data analysis.

\section{Statistical analyses}

All statistical analyses were performed using software (SPSS, V.22.0; SPSS). To adjust for age, gender and the inter-eye correlation from the same participant, the generalised estimating equation (GEE) method was used throughout the analysis whenever applicable. Pearson's correlation was used to assess associations between the OCTA parameters and correlations between OCTA parameters and BCVA after testing by the GEE models. Linear mixed-effects models were used to compare percentage losses of different parameters within one certain diagnostic group. Logistic regression was employed to combine diagnostic parameters into composite diagnostic indices. The area under the receiver operating characteristic curve (AUC) was used to calculate the diagnostic power of the diagnostic parameters. An AUC of 1.0 represents perfect discrimination, whereas an AUC of 0.5 represents accidental discrimination. To compare the diagnostic capabilities of the parameters, the AUCs were compared using the method described by DeLong and colleagues. ${ }^{20} \mathrm{~A} p<0.05$ was considered statistically significant.

\section{RESULTS}

\section{Demographic data}

After excluding poor-quality OCTA images, the study enrolled 208 eyes of 124 subjects with NMOSD (113 eyes with a history 

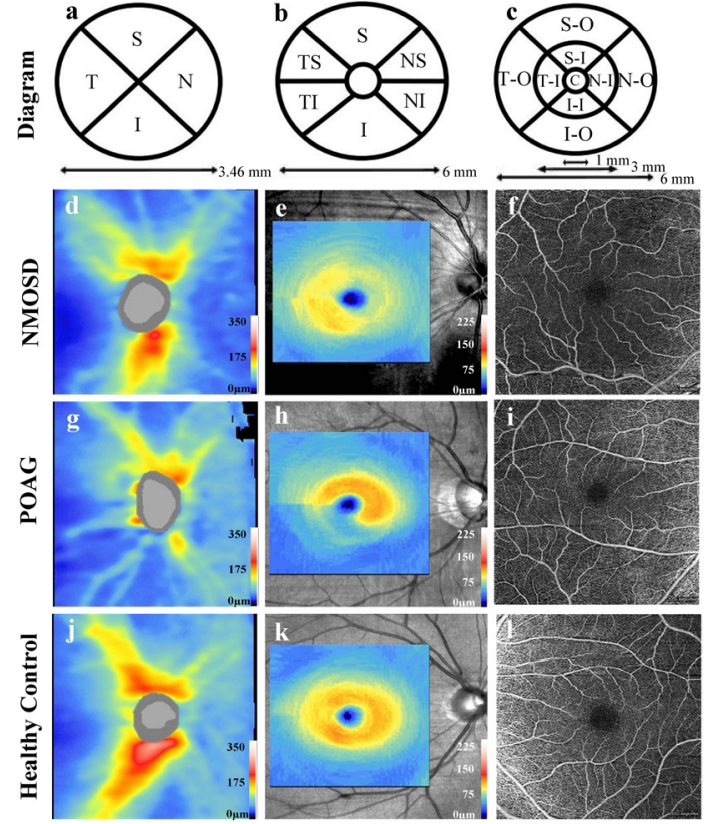

pRNFL thickness

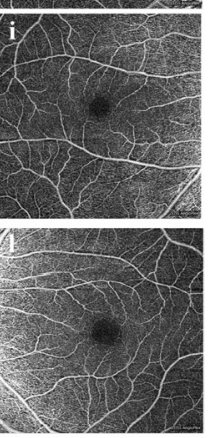

Macular GC-IPL
thickness

Macular microvasculature

Figure 1 Structural and microvasculature measurements in representative eyes. (A, B, C) Diagram of macular and peripapillary measurements using optical coherence tomography angiography. pRNFL thickness was divided into four sectors, macular GC-IPL thickness was divided into six sectors and macular microvasculature was divided into nine sectors. OCTA images of pRNFL thickness, GC-IPL thickness and macular microvasculature: (i) in an eye from a patient with NMOSD ( $D$, $\mathrm{E}, \mathrm{F})$; (ii) in an eye from a patient with POAG $(\mathrm{G}, \mathrm{H}, \mathrm{I})$; and (iii) in an eye from a healthy control (J, K, L). Macular microvasculature was measured

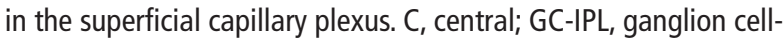
inner plexiform layer; I, inferior; I-I, inferior-inner; I-O, inferior-outer; $\mathrm{N}$, nasal; NI, nasal-inferior; N-I, nasal-inner; NMOSD, neuromyelitis optica spectrum disorder; N-O: nasal-outer; NS, nasal-superior; POAG, primary open angle glaucoma; $p R N F L$, peripapillary retinal nerve fibre layer; $S$, superior; S-I superior-inner; S-O, superior-outer; T, temporal; TI, temporalinferior; T-I, temporal-inner; T-0, temporal-outer; TS, temporal-superior.

of ON, 95 eyes without a history of ON), 124 eyes of 102 subjects with POAG (74 eyes with G-E, 50 eyes with G-A) and 90 eyes of 62 healthy controls. The demographics of the analysed eyes are shown in table 1.

\section{Patterns of losses in macular GC-IPL and microvasculature}

The structural and microvascular parameters were first compared between NMOSD and POAG eyes, using GEE models

to adjust for age, gender and within-subject inter-eye correlations (table 2). The two groups had similar pRNFL thicknesses (NMOSD: $82.28 \pm 19.89 \mu \mathrm{m}, \mathrm{POAG}$ : $74.54 \pm 16.58 \mu \mathrm{m}$, $\mathrm{p}=0.126)$. The NMOSD group showed significant thinning of GC-IPL at the nasal-superior quadrant $(p=0.007)$, whereas the POAG group had a significantly reduced GC-IPL at the inferior and temporal-inferior quadrants $(\mathrm{p}=0.016, \mathrm{p}<0.0001$, respectively). In addition, the NMOSD group showed significantly reduced macular VD and PD compared with the POAG group in all nine quadrants (figure 2A).

As the pRNFL average thickness was also comparable $(\mathrm{p}=0.138)$ between $\mathrm{NMO}+\mathrm{ON}(68.99 \pm 14.27 \mu \mathrm{m})$ and $\mathrm{G}-\mathrm{A}$ $(62.40 \pm 11.23 \mu \mathrm{m})$, we next compared the macular GC-IPL and microvasculature in these two groups (table 2). The thickness of the GC-IPL was significantly reduced in the superior, superiornasal and inferior-nasal quadrants in $\mathrm{NMO}+\mathrm{ON}(\mathrm{p}=0.039$, $\mathrm{p}=0.001, \mathrm{p}=0.007$, respectively), and reduced in the temporalinferior quadrant in G-A ( $\mathrm{p}<0.0001)$, showing significant differences for the quadrant distribution between $\mathrm{NMO}+\mathrm{ON}$ and $\mathrm{G}-\mathrm{A}$ eyes (figure $2 \mathrm{~B}$ ). The macular VD and $\mathrm{PD}$ in all nine quadrants were significantly lower in $\mathrm{NMO}+\mathrm{ON}$ than in $\mathrm{G}-\mathrm{A}$.

To show detailed patterns of losses in each subgroup, the macular GC-IPL, microvascular parameters and pRNFL of NMO$\mathrm{ON}, \mathrm{NMO}+\mathrm{ON}, \mathrm{G}-\mathrm{E}$ and G-A were compared with the healthy control group (figure 2C-F, online supplementary table S1). The order of percentage losses in average GC-IPL thickness was $\mathrm{NMO}+\mathrm{ON}>\mathrm{G}-\mathrm{A}>\mathrm{G}-\mathrm{E}>\mathrm{NMO}-\mathrm{ON}$. The order of percentage losses in whole macular $\mathrm{VD}$ and $\mathrm{PD}$ was $\mathrm{NMO}+\mathrm{ON}>\mathrm{NMO}$ $\mathrm{ON}>\mathrm{G}-\mathrm{A}>\mathrm{G}-\mathrm{E}$. The percentage loss was calculated as $(1-$ (raw measurement $/$ mean value of healthy eyes $)) \times 100(\%)$.

\section{Correlation analysis between structural and angiography parameters}

In each subgroup, VD was strongly correlated with PD (healthy controls: $r=0.994, \mathrm{p}<0.0001 ; \mathrm{G}-\mathrm{E}: \quad r=0.961, \mathrm{p}<0.0001$; G-A: $r=0.975, \mathrm{p}<0.0001 ; \mathrm{NMO}-\mathrm{ON}: r=0.975, \mathrm{p}<0.0001$; $\mathrm{NMO}+\mathrm{ON}: r=0.996, \mathrm{p}<0.0001)$, so we used VD as a representative microvasculature manifestation in the following analysis. Correlation analysis showed that the average thicknesses of GC-IPL were significantly and positively correlated with macular whole VDs in both NMOSD $(r=0.294, \mathrm{p}<0.0001)$ and POAG $(r=0.540, \mathrm{p}<0.0001)$, as well as in each subgroup (NMO-ON: $r=0.224, \mathrm{p}=0.046 ; \mathrm{NMO}+\mathrm{ON}: r=0.217, \mathrm{p}=0.028 ; \mathrm{G}-\mathrm{E}$ : $r=0.490, \mathrm{p}=0.001$; G-A: $r=0.298, \mathrm{p}=0.016$ ).

Correlation analysis between OCTA values and visual function In both disease groups, the BCVA and mean deviation of visual field were significantly correlated with all pRNFL parameters. Among all RNFL parameters, the average pRNFL thickness in

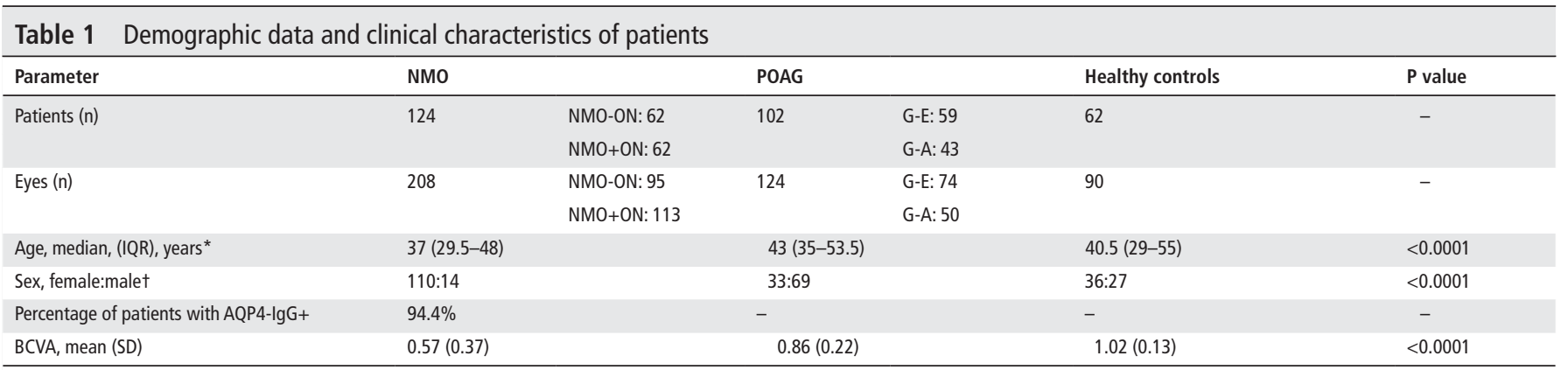

*Kruskal-Wallis test, $p<0.05$ was considered to be statistically significant.

$+\chi^{2}$ test, $p<0.05$ was considered to be statistically significant.

BCVA, best-corrected visual acuity. 
Table 2 Comparison of optical coherence tomography angiography measures after adjusting for age, gender and inter-eye correlation

\begin{tabular}{|c|c|c|c|c|}
\hline \multirow[b]{2}{*}{ OCTA parameters } & \multicolumn{2}{|l|}{ NMOSD vs POAG } & \multicolumn{2}{|l|}{$\mathrm{NMO}+\mathrm{ON}$ vs G-A } \\
\hline & NMOSD value (SD) & POAG value (SD) & $\mathrm{NMO}+\mathrm{ON}$ value $(\mathrm{SD})$ & G-A value (SD) \\
\hline Average pRNFL & $82.28(19.89)$ & $74.54(16.58)$ & $68.99(14.27)$ & $62.40(11.23)$ \\
\hline S pRNFL & $100.14(28.82)$ & $93.41(28.88)$ & $83.40(24.21)$ & $74.26(23.60)$ \\
\hline T pRNFL & $60.64(20.26)$ & $61.28(15.33)$ & $49.23(13.43)$ & $51.90(10.67)$ \\
\hline I pRNFL & $106.31(32.87)^{* * *}$ & $78.71(22.11)$ & $85.93(26.20)^{* * *}$ & $63.42(11.33)$ \\
\hline N pRNFL & $62.28(11.90)^{*}$ & $64.93(11.24)$ & $57.78(10.30)$ & $60.00(9.36)$ \\
\hline Average GC-IPL & $70.26(14.07)$ & $68.26(11.12)$ & $60.79(10.54)$ & 61.18 (8.39) \\
\hline S GC-IPL & $70.80(14.40)$ & $71.52(13.20)$ & $61.49(11.43)^{*}$ & $64.02(11.33)$ \\
\hline TS GC-IPL & $70.26(13.27)$ & $68.34(13.06)$ & $62.11(11.37)$ & $60.28(10.44)$ \\
\hline TI GC-IPL & $71.35(13.26)^{* * *}$ & $60.94(9.72)$ & $63.10(10.96)^{* * *}$ & $54.78(5.37)$ \\
\hline I GC-IPL & $69.47(13.48)^{*}$ & $63.82(9.87)$ & $60.64(10.13)$ & $57.84(6.89)$ \\
\hline NI GC-IPL & $69.41(16.05)$ & $70.90(12.80)$ & $58.35(11.03)^{* *}$ & $63.6(11.05)$ \\
\hline NS GC-IPL & $70.92(16.21)^{* *}$ & $74.64(14.00)$ & $59.95(11.89)^{* *}$ & $67.56(13.50)$ \\
\hline Whole VD & $14.47(3.45)^{* * *}$ & $16.83(1.51)$ & $13.66(3.46)^{* * *}$ & $16.19(1.57)$ \\
\hline Central VD & $5.79(3.33)^{* * *}$ & $8.48(2.91)$ & $5.08(2.84)^{* * *}$ & $8.51(3.10)$ \\
\hline Whole PD & $0.3504(0.0870)^{* * *}$ & $0.4149(0.0384)$ & $0.3295(0.0887)^{* * *}$ & $0.3996(0.0397)$ \\
\hline Central PD & $0.1262(0.0768)^{* * *}$ & $0.1885(0.0666)$ & $0.1094(0.0647)^{* * *}$ & $0.1893(0.0704)$ \\
\hline FAZ area & $0.2473(0.1210)^{* * *}$ & $0.2956(0.1282)$ & $0.2496(0.1257)^{* * *}$ & $0.2718(0.1090)$ \\
\hline
\end{tabular}

OCTA values are presented as mean (SD). VD, PD and FAZ were measured in the superficial capillary plexus. PRNFL and GC-IPL thicknesses are expressed in $\mu \mathrm{m}, \mathrm{VD}$ and PD in $\mathrm{mm}^{-1}$, FAZ area in $\mathrm{mm}^{2}$. ${ }^{*} \mathrm{p}<0.05 ;{ }^{* *} \mathrm{p}<0.01 ;{ }^{* * *} \mathrm{p}<0.001$.

GC-IPL, ganglion cell-inner plexiform layer; FAZ, foveal avascular zone; G-A, advanced glaucoma; I, inferior; N, nasal; NI, nasal-inferior; NMOSD, neuromyelitis optica spectrum disorder; NS, nasal-superior; OCTA optical coherence tomography angiography; ON, optic neuritis; PD, perfusion density; POAG, primary open-angle glaucoma; pRNFL, peripapillary retinal nerve fibre layer; S, superior; T, temporal; TI, temporal-inferior; TS, temporal-superior; $\mathrm{VD}$, vessel density.

the NMOSD and POAG groups $(r=0.558, \mathrm{p}<0.0001 ; r=0.419$, $\mathrm{p}<0.0001$, respectively) showed the highest correlation with BCVA. There were also significant correlations between the

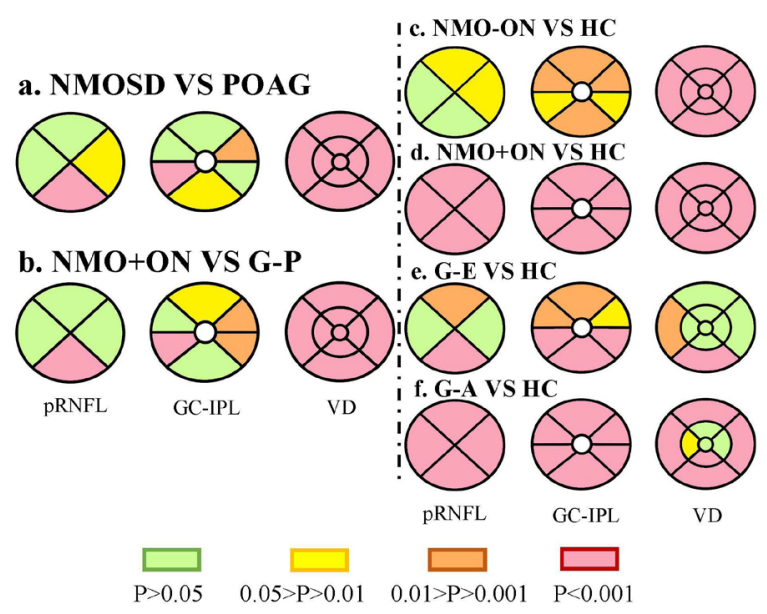

Figure 2 Comparison of structural and angiography parameters between groups. (A) Topographic damage in NMOSD compared with POAG with similar pRNFL average thicknesses. The thickness of the GC-IPL was significantly reduced in the nasal-superior quadrant in NMOSD, and reduced in the inferior and temporal-inferior quadrants in POAG. The NMOSD group showed significantly reduced macular VD compared with the POAG group in all nine quadrants. (B) Topographic damages of $\mathrm{NMO}+\mathrm{ON}$ compared with $\mathrm{G}-\mathrm{A}$ with similar pRNFL average thicknesses. The thickness of the GC-IPL was significantly reduced in the superior, superior-nasal and inferior-nasal quadrants in $\mathrm{NMO+ON}$, and reduced in the temporal-inferior quadrant in G-A. The NMO+ON group showed significantly reduced macular VD compared with G-A in all nine quadrants. (C-F) Topographic damages of NMO-ON, NMO+ON, G-E and G-A compared with healthy controls. GC-IPL, ganglion cell-inner plexiform layer; NMOSD, neuromyelitis optica spectrum disorder; POAG, primary open angle glaucoma; $\mathrm{PRNFL}$, peripapillary retinal nerve fibre layer; VD, vessel density.
BCVA and all GC-IPL parameters, as well as between the mean deviation of the visual field and overall GC-IPL parameters. Among all GC-IPL parameters, the average GC-IPL thickness in the NMOSD and POAG groups $(r=0.530, \mathrm{p}<0.0001 ; r=0.506$, $\mathrm{p}<0.0001$, respectively) showed the highest correlation with BCVA .

For macular VD and PD, we observed significant positive correlations between the BCVA and central, inner and outer segments, and the whole area in both NMOSD and POAG. In NMOSD, there were significant correlations between BCVA and vascular parameters in the central, inferior-inner, inferior-outer, superior-outer and nasal-outer segments. No statistically significant correlations were found between FAZ area and BCVA or FAZ area and mean deviation of visual field in both NMOSD and POAG.

\section{Comparison of percentage losses in macular GC-IPL and VD}

The percentage losses in GC-IPL thickness and VD in each subgroup were compared (online supplementary figure S1). Based on a multivariate analysis, after adjustment for age and sex, the percentage loss in average GC-IPL thickness was significantly more severe than in whole macular VD in the G-E (GCIPL: $9.22 \% \pm 8.26 \%$, VD: $3.47 \% \pm 5.36 \%, \mathrm{p}<0.0001)$ and G-A groups (GC-IPL: $27.96 \% \pm 9.88 \%$, VD: $11.32 \% \pm 8.59 \%$, $\mathrm{p}<0.0001$ ), while whole macula VD was significantly more severe in the NMO-ON group (GC-IPL: $5.19 \% \pm 11.19 \%$, VD: $15.92 \% \pm 16.76 \%, \mathrm{p}<0.0001)$. The percentage losses in GC-IPL and VD were similar in the $\mathrm{NMO}+\mathrm{ON}$ group (GC-IPL: $28.43 \% \pm 11.41 \%$, VD: $25.20 \% \pm 16.94 \%, \mathrm{p}=0.13$ ).

\section{Diagnostic accuracy of OCTA parameters}

The diagnostic capabilities of the OCTA parameters calculated by AUCs are presented in figure 3 and online supplementary table S2. GEE models were used to adjust for age, gender and inter-eye correlation.

To discriminate NMOSD from POAG and healthy controls or to discriminate POAG from NMOSD and healthy controls 


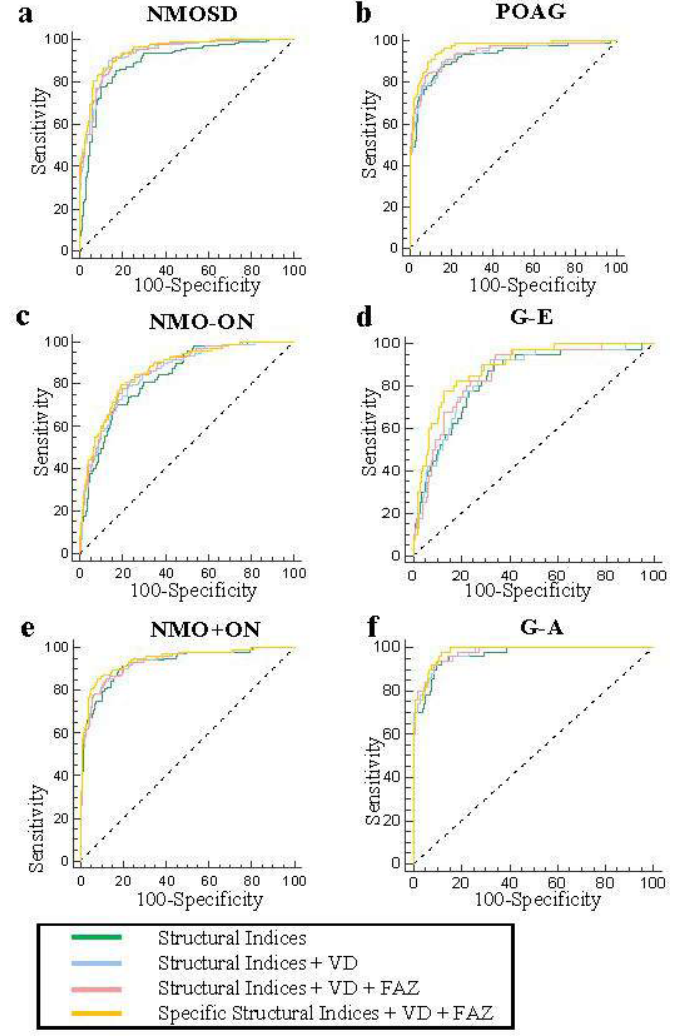

Figure 3 Diagnostic accuracies of optical coherence tomography angiography (OCTA) parameters in discriminating among patients with neuromyelitis optica spectrum disorder (NMOSD), patients with primary open angle glaucoma (POAG) and healthy controls. (A) Distinguishing NMOSD from POAG and healthy controls. In the specific structural optical coherence tomography (OCT) parameters, temporal peripapillary retinal nerve fibre layer (pRNFL) and nasal ganglion cell-inner plexiform layer (GC-IPL) were selected for NMOSD. (B) Distinguishing POAG from NMOSD and healthy controls. In the specific structural OCT parameters, inferior pRNFL and nasal GC-IPL were selected for POAG. (C)

Distinguishing NMO-ON from NMO+ON, G-E, G-A and healthy controls. In the specific structural OCT parameters, inferior pRNFL and nasal GCIPL were selected for the NMO-ON group. (D) Distinguishing G-E from NMO-ON, NMO+ON, G-A and healthy controls. In the specific structural OCT parameters, superior pRNFL and inferior GC-IPL were selected for the G-E group. (E) Distinguishing NMO+ON from NMO-ON, G-E, G-A and healthy controls. In the specific structural OCT parameters, temporal pRNFL and nasal GC-IPL were selected for the NMO+ON group. (F) Distinguishing G-A from NMO-ON, NMO+ON, G-E and healthy controls. In the specific structural OCT parameters, inferior pRNFL and temporal GC-IPL were selected for the G-A group.

(online supplementary table S2a), the structural OCT parameters (pRNFL average thickness, vertical C/D and GC-IPL average thickness) combined with the VD significantly improved diagnostic accuracies compared with only the structural parameters (NMOSD: $p=0.0003$, POAG: $p=0.0354$ ). After selecting the specific quadrants of GC-IPL and pRNFL with the best performance to replace the average thicknesses (temporal pRNFL and nasal GC-IPL were selected for NMOSD; inferior pRNFL and nasal GC-IPL were selected for POAG), the adjusted structural OCT parameters combining the VD and FAZ showed even better diagnostic capability (NMOSD: $p=0.0054$, AUC range $0.917 \sim 0.966$; POAG: $\mathrm{p}=0.0498$, AUC range 0.943 0.989).
For the detection of subgroups (online supplementary table $\mathrm{S} 2 \mathrm{~b}$ ), we quantified the diagnostic capabilities of the OCTA parameters to discriminate one single subgroup from the others. The specific structural OCT parameters modified with quadrant information combining VD and FAZ also showed the best diagnostic capability for the discrimination of all subgroups (NMO+ON: AUC range 0.913 0.963; NMO-ON: AUC range 0.828 0.899; G-A: AUC range 0.961 0.992; G-E: AUC range $0.852 \sim 0.918)$. Among the macular VD parameters, no specific quadrant of VD or PD showed better diagnostic capability than the whole macula VD to discriminate between groups.

\section{FAZ in OCTA as a new biomarker for NMOSD and POAG}

The FAZ area was found to be associated with diagnostic accuracy in patients with POAG (online supplementary table S2a). The FAZ area of eyes with NMOSD, with and without a history of $\mathrm{ON}\left(\mathrm{NMO}+\mathrm{ON}: 0.2496 \pm 0.1257 \mathrm{~mm}^{2}, \mathrm{NMO}-\mathrm{ON}\right.$ : $0.2448 \pm 0.1163 \mathrm{~mm}^{2}$ ), was significantly smaller than that in healthy controls $\left(0.2858 \pm 0.1064 \mathrm{~mm}^{2}\right)$ and POAG eyes (G-A: $0.2718 \pm 0.1090 \mathrm{~mm}^{2}$, G-E: $0.3252 \pm 0.1448 \mathrm{~mm}^{2}$ ). Comparing the binocular FAZ indices of NMOSD patients who experienced monocular ON $(n=28)$, the FAZ area of the eyes with a history of ON (NMO+ON: $0.2450 \pm 0.12039 \mathrm{~mm}^{2}$ ) was smaller than in the contralateral eyes (NMO-ON: $0.2546 \pm 0.12279 \mathrm{~mm}^{2}$ ), but this difference was not statistically significant $(\mathrm{p}=0.352)$.

The size of the FAZ area was found to have a significant negative correlation with central VD in healthy controls $(r=-0.690$, $\mathrm{p}<0.0001), \mathrm{G}-\mathrm{A} \quad(r=-0.505, \mathrm{p}<0.0001), \mathrm{G}-\mathrm{E} \quad(r=-0.485$, $\mathrm{p}=0.002)$ and NMO-ON $(r=-0.227, \mathrm{p}=0.027)$. In the $\mathrm{NMO}+\mathrm{ON}$ group, FAZ area measurement was not correlated with central VD or central PD $(r=-0.029, \mathrm{p}=0.771 ; r=-0.021$, $\mathrm{p}=0.833$, respectively). We did not find any correlation between the FAZ size and the other parameters, including vertical C/D.

\section{DISCUSSION}

NMOSD and POAG are two important causes of registrable blindness worldwide. ${ }^{8} 21$ There is an accumulating body of evidence that the damage seen in the brain and the retina may be driven by common mechanisms in these two diseases. In glaucoma, the responses triggered by axonal injury are likely to be rapidly transmitted both to the retinal ganglion cell body within the inner retina and more distally to the brain, affecting the lateral geniculate nucleus and the visual cortex. ${ }^{8}$ In NMOSD, retrograde transynaptic degeneration has been demonstrated in vivo, implying that abnormalities occurring within the brain could be reflected in the retina, and therefore quantifiable with high-resolution OCT imaging modalities. ${ }^{22-24}$ Numerous studies have shown that both NMOSD and POAG typically result in GC-IPL and pRNFL thinning, indicating the death of a substantial number of retinal ganglion cells and loss of their axons in the optic nerve. ${ }^{5-9}$ In addition, superficial microvascular dropout has been observed in patients with NMOSD and POAG. ${ }^{4} 1125$ Patients with NMOSD and early glaucoma have been investigated separately using OCTA, and both show significantly decreased macular GC-IPL and VD compared with healthy controls. ${ }^{411}$ To the best of our knowledge, however, this is the first study to assess and compare the patterns of structural and microvascular damage seen in eyes with NMOSD and POAG.

We found that the patterns and severity of macular GC-IPL and microvascular loss differed significantly between NMOSD and POAG. GC-IPL thinning was more severe in the superior and nasal quadrants in NMOSD and in the inferior and temporal quadrants in POAG. Patients with NMOSD had a significantly 
lower VD and PD in all quadrants and a decreased size of the FAZ area in comparison with POAG. In addition, we observed that the extent of GC-IPL thinning was more severe than the VD reduction in POAG eyes (both G-E and G-A), while the extent of GC-IPL thinning was less than the VD reduction in the NMOSD group. Our data confirmed the discrimination capability of OCTA by quantifying specific loss patterns in the macular ganglion cells and microvasculature, with the best AUCs above 0.94 for each disease, and above 0.86 for each subgroup. The results of our study further demonstrate that NMOSD and POAG share overlapping vascular changes, ${ }^{26-28}$ although the degree and patterns of changes observed will reflect the different pathophysiology that underlie these two optic neuropathies.

In POAG eyes, the percentage loss in the pRNFL was the greatest, indicating that this area is a prominent injury site. Losses in the macular GC-IPL caused by apoptosis of retinal ganglion cells were then triggered by axon injury, which was rapidly transmitted from the optic disc to the retinal ganglion cell body. ${ }^{8}$ We assumed that the macular microvasculature, which is less sensitive to disease progression than the axons, may reflect secondary retinal degeneration in POAG. The loss of retinal ganglion cells and their axons occurs selectively, with the worst damage in the inferior and temporal quadrants, and the macular microvascular damages start from the inferior-outer and temporal-outer quadrants, which are both coincident with the structural map of the axons and the visual field defects. ${ }^{8}$ In NMOSD eyes, a severe reduction in the macular GC-IPL and microvasculature may be correlated with AQP4-IgG+, given that the fovea is enriched in AQP4-expressing Müller cells, which encompass ganglion cells, contributing to the tight junctions of the blood-brain barrier and playing an important part in the control of osmotic and ionic homeostasis. ${ }^{29-32}$ The ongoing loss of AQP4 results in Müller cell swelling under hypotonic conditions and induces an inflammatory response in the retinal tissues of NMOSD eyes, ${ }^{33}$ during which the macular ganglion cells and microvasculature could be greatly impaired. Subclinical primary retinal pathology in macular foveal thickness, prior to pRNFL thinning, in NMO-ON patients has been demonstrated in a previous study. ${ }^{3}$ However, the exact mechanisms of neuroinflammation, axonal loss and demyelination in NMOSD remain unknown. ${ }^{32}$ We speculate that these pathological changes in the macula including GP-IPL structure and microvasculature indicate primary sites of injury, while thinning of the pRNFL represents secondary retinal degeneration in NMOSD. The pathophysiology of the distinct distribution of GC-IPL thinning in the nasal and superior quadrants deserves further evaluation. The nasal preponderance of GC-IPL damage in NMOSD might be related to a greater sensitivity to injury in the papillomacular bundle, which has been observed in patients with multiple sclerosis presenting with ON. ${ }^{34} 35$ As expected, there was a significant correlation between BCVA and both pRNFL and GC-IPL parameters in the NMOSD and POAG disease groups.

The FAZ, which is a small region devoid of retinal vessels in the centre of the fovea, has been quantified in other neurodegenerative diseases, such as Alzheimer disease and Parkinson disease to determine its relevance to the pathology and disease progression. ${ }^{36}{ }^{37}$ Patients with Alzheimer disease $(\mathrm{n}=27$ eyes) had the largest mean FAZ area, which was significantly different from that of POAG ( $\mathrm{n}=27$ eyes) $(\mathrm{p}<0.001)$ as well as healthy controls $\left(\mathrm{n}=27\right.$ eyes) $(\mathrm{p}<0.001) .{ }^{36}$ In contrast, the FAZ area in Parkinson disease $(n=25$ eyes) was significantly smaller compared with healthy controls $\left(\mathrm{n}=17\right.$ eyes) $(\mathrm{p}=0.02) .{ }^{37}$ Our findings provide new information regarding the FAZ measurements in eyes with NMOSD ( $\mathrm{n}=208$ eyes). The FAZ size was significantly reduced in eyes with NMOSD compared with both healthy controls $(\mathrm{n}=90$ eyes) and POAG eyes $(\mathrm{n}=124$ eyes). Moreover, the percentage loss in the FAZ was comparable with that in the GC-IPL and it was significantly greater than the pRNFL in eyes with NMO-ON. This finding suggests that FAZ may be a novel and sensitive biomarker of retinal neuron loss in NMOSD. The reasons for the lengthening of foveal capillaries and the smaller FAZ area in NMOSD remain to be clarified. Although the AQP4-expressing Müller cells in FAZ may play an aetiological role in vascular remodelling in NMOSD, additional studies are required to quantify capillary remodelling and to demonstrate whether cell loss or damage leads to vasculogenesis from the surrounding capillaries. Our findings need independent replication and further studies with repeated measurements over a longer follow-up period will provide more detailed information about the chronology and changes in FAZ metrics in NMOSD. In addition, reliable OCTA is only possible in regions where there is a strong signal. Therefore, OCTA cannot detect flow in low signal regions even if it is present. As blood flow in ocular vessels of NMOSD is slow, the vessels may be silent on OCTA, creating a false-negative appearance even if they are present.

In summary, this is the first study comparing macular GC-IPL and microvasculature between patients with NMOSD compared with POAG and healthy controls. The results showed that NMOSD and POAG are associated with specific patterns of retinal structural and microvasculature dysfunction reflecting their different pathogenesis. FAZ area in NMOSD represents an attractive disease biomarker that could be applied to monitoring disease progression and as an outcome measure in clinical trials investigating novel immunomodulatory agents.

\section{Author affiliations}

${ }^{1}$ State Key Laboratory of Ophthalmology, Zhongshan Ophthalmic Center, Sun Yat-sen University, Guangzhou, China

${ }^{2}$ Department of Neurology, Third Affiliated Hospital, Sun Yat-Sen University, Guangzhou, China

${ }^{3}$ Department of Molecular and Cellular Pharmacology, University of Miami Miller School of Medicine, Miami, Florida, USA

${ }^{4}$ Sylvester Comprehensive Cancer Center, University of Miami Miller School of

Medicine, Miami, Florida, USA

${ }^{5}$ Cambridge Centre for Brain Repair and MRC Mitochondrial Biology Unit,

Department of Clinical Neurosciences, University of Cambridge, Cambridge, UK

${ }^{6}$ Cambridge Eye Unit, Addenbrooke's Hospital, Cambridge University Hospitals, Cambridge, UK

${ }^{7}$ Moorfields Eye Hospital, London, United Kingdom

${ }^{8} \mathrm{UCL}$ Institute of Ophthalmology, University College London, London, United Kingdom

${ }^{9}$ Singapore National Eye Center, Duke-NUS Medical School, Singapore

${ }^{10}$ Center of Precision Medicine, Sun Yat-sen University, Guangzhou, China

Contributors $\mathrm{XZ}, \mathrm{HX}, \mathrm{CL}$ and LZ contributed equally to the study. Conception and design: HL, HX and WQ. Analysis and interpretation: XZ, LZ and HX. Data collection: $C L, H X, H L, J W, X Z, J W$ and RW. Critically revised the manuscript: PY-W-M, DST, $H X, Y Z, C C, X L, D L, X W$ and WQ. Obtained funding: $H L, H X$ and PY-W-M. Overall responsibility: $H L, W Q$ and $X L$.

Funding $\mathrm{HL}$ was funded by the National Key R \& D Program of China (2018YFC0116500), the National Natural Science Foundation of China (81770967, $81822010)$ and the Science and Technology Planning Projects of Guangdong Province (2018B010109008). HX was funded by the Natural Science Foundation of Guangdong Province (2017A030313649). PY-W-M was supported by a Clinician Scientist Fellowship Award (G1002570) from the Medical Research Council

(UK), and also received funding from Fight for Sight (UK), the Isaac Newton Trust (UK), the National Eye Research Centre (UK), the UK National Institute of Health Research (NIHR) as part of the Rare Diseases Translational Research Collaboration, and the NIHR Biomedical Research Centre based at Moorfields Eye Hospital NHS Foundation Trust and UCL Institute of Ophthalmology. The views expressed are those of the authors and not necessarily those of the NHS, the NIHR or the Department of Health.

Disclaimer The funders had no role in the study design, data collection, data analysis, data interpretation or writing of the report. 
Competing interests None declared.

Patient consent for publication Obtained.

Ethics approval This study was approved by the Ethics Committee of Zhongshan Ophthalmic Center, Sun Yat-sen University and it was conducted in accordance with the tenets of the Declaration of Helsinki.

Provenance and peer review Not commissioned; externally peer reviewed.

Data availability statement Data are available on reasonable request. Basic information and OCTA data collected from participants are kept in reserve in Science and Technology Office, Zhongshan Ophthalmic Center, Sun Yat-sen University. HL ( gddlht@aliyun.com) is responsible for any detailed inquiry. Protocols and statistical analysis plans have been submitted to Ethics Committee of Zhongshan Ophthalmic Center, Sun Yat-sen University.

Open access This is an open access article distributed in accordance with the Creative Commons Attribution Non Commercial (CC BY-NC 4.0) license, which permits others to distribute, remix, adapt, build upon this work non-commercially, and license their derivative works on different terms, provided the original work is properly cited, appropriate credit is given, any changes made indicated, and the use is non-commercial. See: http://creativecommons.org/licenses/by-nc/4.0/.

ORCID iDs

Daniel S Ting http://orcid.org/0000-0003-2264-7174

Haotian Lin http://orcid.org/0000-0003-4672-9721

\section{REFERENCES}

1 Kim S-H, Kim W, Li XF, et al. Clinical spectrum of CNS aquaporin-4 autoimmunity. Neurology 2012;78:1179-85.

2 Cree BAC, Bennett JL, Kim HJ, Bruce AC, Jeffrey LB, JK H, et al. Inebilizumab for the treatment of neuromyelitis optica spectrum disorder (N-MOmentum): a double-blind, randomised placebo-controlled phase 2/3 trial. Lancet 2019;394:10206

3 Jeong $\mathrm{IH}$, Kim HJ, Kim N-H, et al. Subclinical primary retinal pathology in neuromyelitis optica spectrum disorder. J Neurol 2016;263:1343-8.

4 Kwapong WR, Peng C, He Z, et al. Altered macular microvasculature in neuromyelitis optica spectrum disorders. Am J Ophthalmol 2018;192:47-55.

5 Merle $\mathrm{H}$, Olindo S, Donnio A, et al. Retinal peripapillary nerve fiber layer thickness in neuromyelitis optica. Invest Ophthalmol Vis Sci 2008:49:4412-7.

6 Lange AP, Sadjadi R, Zhu F, et al. Spectral-Domain optical coherence tomography of retinal nerve fiber layer thickness in NMO patients. J Neuroophthalmol 2013;33:213-9

7 Naismith RT, Tutlam NT, Xu J, et al. Optical coherence tomography differs in neuromyelitis optica compared with multiple sclerosis. Neurology 2009;72:1077-82.

8 Jonas JB, Aung T, Bourne RR, et al. Glaucoma. Lancet 2017;390:2183-93.

9 Weinreb RN, Aung T, Medeiros FA. The pathophysiology and treatment of glaucoma: a review. JAMA 2014;311:1901-11.

10 Peng A, Qiu X, Zhang L, et al. Evaluation of the retinal nerve fiber layer in neuromyelitis optica spectrum disorders: a systematic review and meta-analysis. J Neurol Sci 2017:383:108-13.

11 Hou H, Moghimi S, Zangwill LM, et al. Macula vessel density and thickness in early primary open-angle glaucoma. Am J Ophthalmol 2019;199:120-32.

12 Hollands $\mathrm{H}$, Johnson $\mathrm{D}$, Hollands $\mathrm{S}$, et al. Do findings on routine examination identify patients at risk for primary open-angle glaucoma? the rational clinical examination systematic review. JAMA 2013;309:2035-42.

13 Rao HL, Addepalli UK, Chaudhary S, et al. Ability of different scanning protocols of spectral domain optical coherence tomography to diagnose preperimetric glaucoma. Invest Ophthalmol Vis Sci 2013;54:7252-7.

14 Gabilondo I, Martínez-Lapiscina EH, Fraga-Pumar E, et al. Dynamics of retinal injury after acute optic neuritis. Ann Neurol 2015;77:517-28.
15 Rebolleda G, Sánchez-Sánchez C, González-López JJ, et al. Papillomacular bundle and inner retinal thicknesses correlate with visual acuity in nonarteritic anterior ischemic optic neuropathy. Invest Ophthalmol Vis Sci 2015;56:682-92.

16 Ohayon A, Sacconi R, Semoun 0, et al. Choroidal neovascular area and vessel density comparison between two SWEPT-SOURCE optical coherence tomography angiography devices. Retina 2020:40:521-8.

17 Spain RI, Liu L, Zhang X, et al. Optical coherence tomography angiography enhances the detection of optic nerve damage in multiple sclerosis. Br J Ophthalmol 2018;102:520-4

18 Wingerchuk DM, Banwell B, Bennett JL, et al. International consensus diagnostic criteria for neuromyelitis optica spectrum disorders. Neurology 2015;85:177-89.

19 Waters PJ, McKeon A, Leite MI, et al. Serologic diagnosis of NMO: a multicenter comparison of aquaporin-4-IgG assays. Neurology 2012;78:665-71.

20 DeLong ER, DeLong DM, Clarke-Pearson DL. Comparing the areas under two or more correlated receiver operating characteristic curves: a nonparametric approach. Biometrics 1988;44:837-45.

21 Papp V, Illes Z, Magyari M, et al. Nationwide prevalence and incidence study of neuromyelitis optica spectrum disorder in Denmark. Neurology 2018;91:e227 5:e2265-75.

22 Banker $\mathrm{P}$, Sonni S, Kister I, et al. Pencil-thin ependymal enhancement in neuromyelitis optica spectrum disorders. Mult Scler 2012;18:1050-3.

23 Ayrignac X, Dalière CC, Nerrant E, et al. Extensive cerebral white matter involvement in a patient with NMO spectrum disorder. Mult Scler 2014:20:1401-3.

24 Chanson J-B, Lamy J, Rousseau F, et al. White matter volume is decreased in the brain of patients with neuromyelitis optica. Eur J Neurol 2013;20:361-7.

25 Huang Y, Zhou L, ZhangBao J, et al. Peripapillary and parafoveal vascular network assessment by optical coherence tomography angiography in aquaporin-4 antibodypositive neuromyelitis optica spectrum disorders. Br J Ophthalmol 2019;103:789-96.

26 Lefkowitz D, Angelo JN. Neuromyelitis optica with unusual vascular changes. Arch Neurol 1984;41:1103-5.

27 Lucchinetti CF, Mandler RN, McGavern D, et al. A role for humoral mechanisms in the pathogenesis of Devic's neuromyelitis optica. Brain 2002;125:1450-61.

28 Anderson DR, Glaucoma ADR. Glaucoma, capillaries and pericytes. 1. blood flow regulation. Ophthalmologica 1996;210:257-62.

29 landiev I, Pannicke T, Biedermann B, et al. Ischemia-Reperfusion alters the immunolocalization of glial aquaporins in rat retina. Neurosci Lett 2006:408:108-12.

30 Nagelhus EA, Mathiisen TM, Ottersen OP. Aquaporin-4 in the central nervous system: cellular and subcellular distribution and coexpression with Kir4.1. Neuroscience 2004;129:905-13.

31 Bringmann A, Pannicke T, Grosche J, et al. Müller cells in the healthy and diseased retina. Prog Retin Eye Res 2006;25:397-424.

32 You Y, Zhu L, Zhang T, et al. Evidence of Müller glial dysfunction in patients with aquaporin-4 immunoglobulin G-Positive neuromyelitis optica spectrum disorder. Ophthalmology 2019;126:801-10.

33 Pannicke T, Wurm A, landiev I, et al. Deletion of aquaporin-4 renders retinal glial cells more susceptible to osmotic stress. J Neurosci Res 2010;88:n/a-88.

34 Green AJ, Cree BAC. Distinctive retinal nerve fibre layer and vascular changes in neuromyelitis optica following optic neuritis. J Neurol Neurosurg Psychiatry 2009;80:1002-5.

35 Manogaran P, Traboulsee AL, Lange AP. Longitudinal study of retinal nerve fiber layer thickness and macular volume in patients with neuromyelitis optica spectrum disorder. J Neuroophthalmol 2016;36:363-8.

36 Zabel P, Kaluzny JJ, Wilkosc-Debczynska M, et al. Comparison of retinal microvasculature in patients with Alzheimer's disease and primary open-angle glaucoma by optical coherence tomography angiography. Invest Ophthalmol Vis Sci 2019;60:3447-55.

37 Miri S, Shrier EM, Glazman S, et al. The avascular zone and neuronal remodeling of the fovea in Parkinson disease. Ann Clin Transl Neurol 2015;2:196-201. 